\title{
Genomic expression profiling and bioinformatics analysis on diabetic nephrology with ginsenoside $\mathbf{R g 3}$
}

\author{
JUAN WANG ${ }^{1}$, CHUNLI CUI ${ }^{2}$, LI FU $^{3}$, ZILI XIAO ${ }^{4}$, NANZI XIE ${ }^{1}$, YANG LIU $^{1}$, \\ LU YU $^{1}$, HAIFENG WANG ${ }^{1}$ and BANGZHEN LUO ${ }^{1}$ \\ Departments of ${ }^{1}$ Geriatric and ${ }^{2}$ Nephrology, Shanghai Tongji Hospital Affiliated to Tongji University, \\ Shanghai 200065; ${ }^{3}$ Dalian Fusheng Natural Pharmaceutical Development Co. Ltd., Dalian, Liaoning 116600; \\ ${ }^{4}$ Shanghai Jinfang Biotechnology Co. Ltd., Shanghai 201210, P.R. China
}

Received May 14, 2015; Accepted April 19, 2016

DOI: $10.3892 / \mathrm{mmr} .2016 .5349$

\begin{abstract}
Diabetic nephropathy (DN), a common diabetes-related complication, is the leading cause of progressive chronic kidney disease (CKD) and end-stage renal disease. Despite the rapid development in the treatment of DN, currently available therapies used in early DN cannot prevent progressive CKD. The exact pathogenic mechanisms and the molecular events underlying DN development remain unclear. Ginsenoside $\operatorname{Rg} 3$ is a herbal medicine with numerous pharmacological effects. To gain a greater understanding of the molecular mechanism and signaling pathway underlying the effect of ginsenoside Rg3 in DN therapy, an RNA sequencing approach was performed to screen differential gene expression in a rat model of DN treated with ginsenoside $\mathrm{Rg} 3$. A combined bioinformatics analysis was then conducted to obtain insights into the underlying molecular mechanisms of the disease development, in order to identify potential novel targets for the treatment of DN. Six Sprague-Dawley male rats were randomly divided into 3 groups: Normal control group, DN group and ginsenoside- $\mathrm{Rg} 3$ treatment group, with two rats in each group. RNA sequencing was adopted for transcriptome profiling of cells from the renal cortex of DN rat model. Differentially expressed genes were screened out. Cluster analysis, Gene Ontology (GO) and Kyoto Encyclopedia of Genes and Genomes (KEGG) enrichment analysis were used to analyze the differentially expressed genes. In total, 78 differentially expressed genes in the DN control group were identified when compared with the normal control group, of which 52 genes were
\end{abstract}

Correspondence to: Dr Chunli Cui, Department of Nephrology, Shanghai Tongji Hospital Affiliated to Tongji University, 389 Xincun Road, Putuo, Shanghai 200065, P.R. China

E-mail: tjchunlicui@126.com

Mr. Li Fu, Dalian Fusheng Natural Pharmaceutical Development Co. Ltd., 5 Tieshan Road, Dalian, Liaoning 116600, P.R. China

E-mail: lifudalian@126.com

Key words: diabetic nephropathy, ginsenoside Rg3, RNA-Seq, bioinformatics analysis upregulated and 26 genes were downregulated. Differential expression of 43 genes was observed in the ginsenoside-Rg3 treatment group when compared with the DN control group, consisting of 10 upregulated genes and 33 downregulated genes. Notably, 21 that were downregulated in the DN control group compared with the control were then shown to be upregulated in the ginsenoside- $\mathrm{Rg} 3$ treatment group compared with the DN control group. In addition, 7 upregulated genes in the DN control group compared with the control were then shown to be downregulated in the ginsenoside- $\operatorname{Rg} 3$ treatment group compared with the DN control group. Cluster analysis based on differentially expressed genes indicated that the transcriptomes are quite different among the samples. Distinct GO terms associated with these groups of genes were shown to be enriched. KEGG pathway analysis demonstrated that differentially expressed genes were predominantly involved in the fatty acid metabolism pathway and peroxisome proliferator-activated receptor (PPAR) signaling pathway. To the best of our knowledge, this study was the first to present whole genome expression profiling in DN with ginsenoside-Rg3 treatment by RNA-Seq. A set of differentially expressed genes and pathways were identified. These data provided an insight into understanding the molecular mechanisms underlying the effect of ginsenoside- $\mathrm{Rg} 3$ treatment of $\mathrm{DN}$.

\section{Introduction}

Diabetic nephropathy (DN) is a chronic microvascular complication of type 1 and type 2 diabetes mellitus and is the leading cause of progressive chronic kidney disease and end-stage renal disease, which puts a serious burden on the patient's family and on society (1).

The exact pathogenic mechanisms and the molecular events of DN remain unclear. Evidence suggests that environmental and genetic factors are involved in the development of DN (2). A number of factors are known to be critical in the development of DN, such as insulin-like growth factor 1 (IGF1), transforming growth factor beta, platelet-derived growth factor (PDGF), fibroblast growth factor (FGF), interleukins (ILs), tumor necrosis factor- $\alpha$ (TNF- $\alpha$ ), vascular endothelial growth factor (VEGF) and endothelin (3). These factors effect processes including glucose metabolism, renal hemodynamics, 
cell matrix metabolism, cell proliferation, cell hypertrophy, cell apoptosis, abnormal angiogenesis and cytokine-mediated inflammatory response, which are involved in DN pathogenesis. Searching for agents that regulate the expression of these cytokines to intervene and prevent the development of DN an area of research.

Ginsenoside $\mathrm{Rg} 3$ is an active ingredients in Ginseng, which can inhibit the growth and metastasis of tumors through the downregulation of VEGF, bFGF gene expression and inhibiting the formation of abnormal new blood vessels. Besides, ginsenoside $\operatorname{Rg} 3$ has a number of pharmacological effects, including the inducing the apoptosis of tumor cells, promoting $\mathrm{T}$ lymphocyte mitosis and NK cell activity, stimulating the phagocytic function of the reticuloendothelial system, promoting the secretion of B lymphocyte antibodies, promoting antiplatelet aggregation to prevent thrombosis, dilating the blood vessels and increasing blood supply $(4,5)$.

Therefore, it was hypothesized that ginsenoside $\mathrm{Rg} 3$ could prevent the occurrence and development of DN by effecting the gene expression and regulation of various cytokines to delay the progression of DN to end-stage renal failure. To the best of our knowledge, this study was the first to observe the effect of ginsenoside $\operatorname{Rg} 3$ on the changes of the gene expression profile in DN rats, which may aid in the identification of novel targets for the treatment of DN.

\section{Materials and methods}

Animal group. Six male Sprague-Dawley (SD) rats were provided by Shanghai Laboratory Animal Center (Shanghai, China) (average weight, $300 \mathrm{~g}$ ). The rats were divided randomly into three groups: Control group (sample ID: 1 and 3), DN control group (sample ID: 8 and 9) and ginsenoside-Rg3 treatment group (sample ID: 15 and 16), with two rats in each group.

Modeling and Rg3 drugs. The normal control group was given normal feed. The DN control and ginsenoside-Rg3 treatment groups were administered a high lipid diet for two months, then were treated with intraperitoneal injection of streptozotocin (STZ; $45 \mathrm{mg} / \mathrm{kg}$ ) induced diabetic nephropathy. After the models were established, the ginsenoside-Rg3 treatment group were administered $\operatorname{Rg} 3(0.5 \mathrm{mg} / \mathrm{kg})$ once a day for 30 days.

RNA extraction. Samples from renal cortex were collected and stored in liquid nitrogen at $-80^{\circ} \mathrm{C}$ until RNA extraction was performed. Total RNA was extracted from the samples of renal cortex using TRIzol reagent (Thermo Fisher Scientific, Inc., Waltham, MA, USA) according to the manufacturer's instructions. RNA quantity and quality were measured using the NanoDrop ND-1000 spectrophotometer and Agilent Bioanalyzer 2100 (Agilent technologies, Santa Clara, CA, USA).

RNA-seq data generation. RNA-seq libraries were prepared in accordance with Illumina's sample preparation protocol. The libraries were sequenced onto an Illumina HiSeq2000 instrument (Illumina Inc., San Diego, CA, USA) and subjected to 100 cycles of paired end ( $2 \times 100 \mathrm{bp})$ sequencing. The processing
Table I. Top ten enriched differentially expressed genes in the DN control group compared with the normal control group.

\begin{tabular}{|c|c|}
\hline Gene & Description \\
\hline \multicolumn{2}{|l|}{ Upregulated } \\
\hline Angptl4 & Angiopoietin-like 4 \\
\hline Hmgcs 2 & $\begin{array}{l}\text { 3-Hydroxy-3-methylglutaryl-CoA } \\
\text { synthase } 2 \text { (mitochondrial) }\end{array}$ \\
\hline Haver1 & Hepatitis A virus cellular receptor 1 \\
\hline Gpx2 & Glutathione peroxidase 2 \\
\hline Slc $25 \mathrm{a} 25$ & $\begin{array}{l}\text { Solute carrier family } 25 \text { (mitochondrial } \\
\text { carrier, phosphate carrier), member } 25\end{array}$ \\
\hline Rarres2 & $\begin{array}{l}\text { Retinoic acid receptor responder } \\
\text { (tazarotene induced) } 2\end{array}$ \\
\hline Spp1 & Secreted phosphoprotein 1 \\
\hline Serhl2 & Serine hydrolase-like 2 \\
\hline Ecil & Enoyl-CoA $\Delta$ isomerase 1 \\
\hline Apoc2 & Apolipoprotein C-II \\
\hline \multicolumn{2}{|l|}{ Downregulated } \\
\hline LOC361914 & $\begin{array}{l}\text { Similar to solute carrier family } 7 \text { (cationic } \\
\text { amino acid transporter, y+system), } \\
\text { member } 12\end{array}$ \\
\hline Cacng5 & $\begin{array}{l}\text { Calcium channel, voltage-dependent, } \\
\gamma \text { subunit } 5\end{array}$ \\
\hline Slc7a12 & $\begin{array}{l}\text { Solute carrier family } 7 \text { (cationic amino acid } \\
\text { transporter, y+system), member } 12\end{array}$ \\
\hline LOC686288 & Similar to olfactory receptor Olr1668 \\
\hline Prima 1 & Proline rich membrane anchor 1 \\
\hline Slco1a6 & $\begin{array}{l}\text { Solute carrier organic anion transporter } \\
\text { family, member } 1 \mathrm{a} 6\end{array}$ \\
\hline RT1-CE1 & RT1 class I, locus 1 \\
\hline Hnmt & histamine N-methyltransferase \\
\hline Cyp2c11 & $\begin{array}{l}\text { Cytochrome P } 450 \text {, subfamily } 2 \text {, } \\
\text { polypeptide } 11\end{array}$ \\
\hline Cyp2c24 & $\begin{array}{l}\text { Cytochrome P } 450 \text {, family } 2 \text {, subfamily c, } \\
\text { polypeptide } 24\end{array}$ \\
\hline
\end{tabular}

of fluorescent images into sequences, base-calling and quality value calculations were performed using the Illumina data processing pipeline (version 1.8). Prior to assembly, high-quality clean reads were generated using FASTX toolkit pipeline (version 0.0.13), then the resulting high-quality reads were mapped onto the UCSC (mm10) using Tophat (version: 2.0.6) (6). Cufflink (version: 2.0.2) (7) was used to process the Tophat alignments. Additionally, transcript expression levels were estimated using Fragments Per Kilobases per Million reads (FPKM) values. Finally, the program Cuffdiff was used to define differentially expressed genes as a gene set for further analysis. The selection criteria of differentially expressed genes was based upon the fold-changes of the expression levels $(\mathrm{P}<0.05)$. If the gene expression status was consistent in two comparison groups (DN control group vs. the normal control group including sample ID1 vs. ID8 and sample ID3 vs. ID9; ginsenoside- $\operatorname{Rg} 3$ treatment group vs. DN control group including sample ID8 vs. ID15 and sample ID9 vs. ID16), they were defined as differentially 
Table II. Top ten enriched differentially expressed genes in the ginsenoside-Rg3 treatment group compared with the DN control group.

\begin{tabular}{|c|c|}
\hline Gene & Description \\
\hline \multicolumn{2}{|l|}{ Upregulated } \\
\hline Cyp2c24 & $\begin{array}{l}\text { Cytochrome P } 450 \text {, family } 2 \text {, subfamily c, } \\
\text { polypeptide } 24\end{array}$ \\
\hline Gtpbp4 & GTP binding protein 4 \\
\hline Prima1 & Proline rich membrane anchor 1 \\
\hline Slcola6 & $\begin{array}{l}\text { Solute carrier organic anion transporter } \\
\text { family, meber } 1 \mathrm{a} 6\end{array}$ \\
\hline Prlr & Prolactin receptor \\
\hline Tmem72 & Transmembrane protein 72 \\
\hline Acadsb & $\begin{array}{l}\text { Acyl-CoA dehydrogenase, short/branched } \\
\text { chain }\end{array}$ \\
\hline Ppm1k & $\begin{array}{l}\text { Protein phosphatase, } \mathrm{Mg}^{2+} / \mathrm{Mn}^{2+} \text { dependent, } \\
1 \mathrm{~K}\end{array}$ \\
\hline LOC100365744 & Hypothetical protein LOC 100365744 \\
\hline Slc30a1 & $\begin{array}{l}\text { Solute carrier family } 30 \text { (zinc } \\
\text { transporter, membrane } 1\end{array}$ \\
\hline \multicolumn{2}{|l|}{ Downregulated } \\
\hline Havcr1 & Hepatitis A virus cellular receptor 1 \\
\hline Ril & Reversion induced LIM gene \\
\hline Hmgcs 2 & $\begin{array}{l}\text { 3-Hydroxy-3-methylglutaryl-CoA } \\
\text { synthase } 2 \text { (mitochondrial) }\end{array}$ \\
\hline Slc25a25 & $\begin{array}{l}\text { Solute carrier family } 25 \text { (mitochondrial } \\
\text { carrier, phosphate carrier), member } 25\end{array}$ \\
\hline Cxcl11 & Chemokine (C-X-C motif) ligand 11 \\
\hline Acaa1b & Acetyl-Coenzyme A acyltransferase 1B \\
\hline Resp18 & Regulated endocrine-specific protein 18 \\
\hline Rarres2 & $\begin{array}{l}\text { Retinoic acid receptor responder } \\
\text { (tazarotene induced) } 2\end{array}$ \\
\hline Rbp1 & Retinol binding protein 1 , cellular \\
\hline Gdf15 & Growth differentiation factor 15 \\
\hline
\end{tabular}

expressed genes. All analyses were performed at Shanghai Biotechnology Corporation (Shanghai, China).

Gene Ontology (GO) and Kyoto Encyclopedia of Genes and Genomes (KEGG) enrichment analyses. In order to examine the biological significance of the differentially expressed genes, the GO and KEGG (http://www.genome.jp/kegg/) pathway analysis produced by Kanehisa Laboratories (Kyoto, Japan) were performed to investigate their functional and pathway annotation $(8,9)$. GO and pathway analyses were conducted using TargetMine (available at http://targetmine. nibio.go.jp/) based on a hypergeometric test (10).

\section{Results}

\section{Differentially expressed genes generated by RNA-Seq}

Normal control group and DN control group. Totally, 78 differentially expressed genes in the DN control group were identified compared with the normal control group. Of
Table III. Twenty-eight differentially expressed genes.

Gene name Description

LOC100365744 Hypothetical protein LOC100365744

Ppm1k

Acadsb

Protein phosphatase $\mathrm{Mg}^{2+} / \mathrm{Mn}^{2+}$ dependent, $1 \mathrm{~K}$

Slcola6

Prlr

Cyp2c24

Prima1

Hmgcs2

Acyl-CoA dehydrogenase, short/branched chain

Solute carrier organic anion transporter family, member $1 \mathrm{a} 6$

Prolactin receptor

Cytochrome P450, family 2, subfamily c, polypeptide 24

3-hydroxy-3-methylglutaryl-CoA

Eci1

Gdf15

Plin2

Slc25a25

Proline rich membrane anchor 1

synthase 2 (mitochondrial)

Enoyl-CoA $\Delta$ isomerase 1

Growth differentiation factor 15

Perilipin 2

Haver1

Acaa1a

Ephx1

Pck1

Solute carrier family 25 (mitochondrial carrier, phosphate carrier), member 25

Hepatitis A virus cellular receptor 1

Acetyl-Coenzyme A acyltransferase 1A

Epoxide hydrolase 1, microsomal

Phosphoenolpyruvate carboxykinase

1 (soluble)

Car15

Carbonic anhydrase 15

Apoc3

Ddit41

Ril

Apolipoprotein C-III

Cyp4a1

DNA-damage-inducible transcript 4-like

Reversion induced LIM gene

Cytochrome P450, family 4,

subfamily a, polypeptide 1

Rbp1 Retinol binding protein 1, cellular

Mt1a Metallothionein 1a

Car4

Carbonic anhydrase 4

Glutathione peroxidase 1

Betaine-homocysteine

S-methyltransferase

Rarres2 Retinoic acid receptor responder

(tazarotene induced) 2

Resp18

Regulated endocrine-specific protein 18

these, 52 genes were upregulated while 26 genes were downregulated. The top ten enriched differentially expressed genes based on fold-change are listed in Table I.

DN control group and ginsenoside-Rg3 treatment group. In total, 43 differentially expressed genes in the ginsenoside-Rg3 treatment group were identified compared with the DN control group. Of these, 10 genes were upregulated while 33 genes were downregulated. The top ten enriched differentially expressed genes based on fold-change are listed in Table II.

Bioinformatics analysis. In total, 21 genes that were downregulated in the DN control group compared with the 


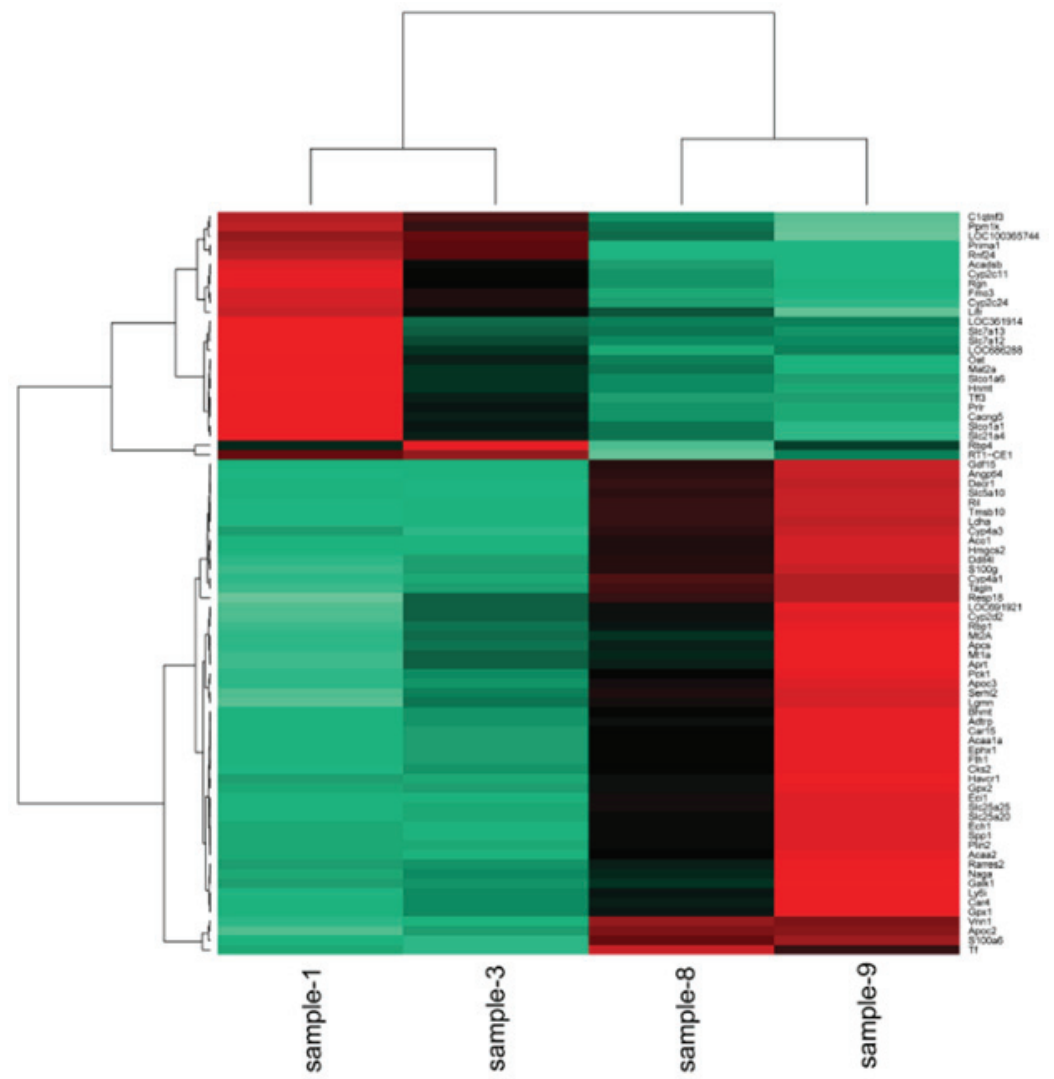

Figure 1. Hierarchical clustering of differentially expressed genes in the DN control group compared with the normal control group. Each row represents a differentially expressed gene, while each column represents a sample. In the heatmap, green represents genes that are downregulated and red represents genes that are upregulated.

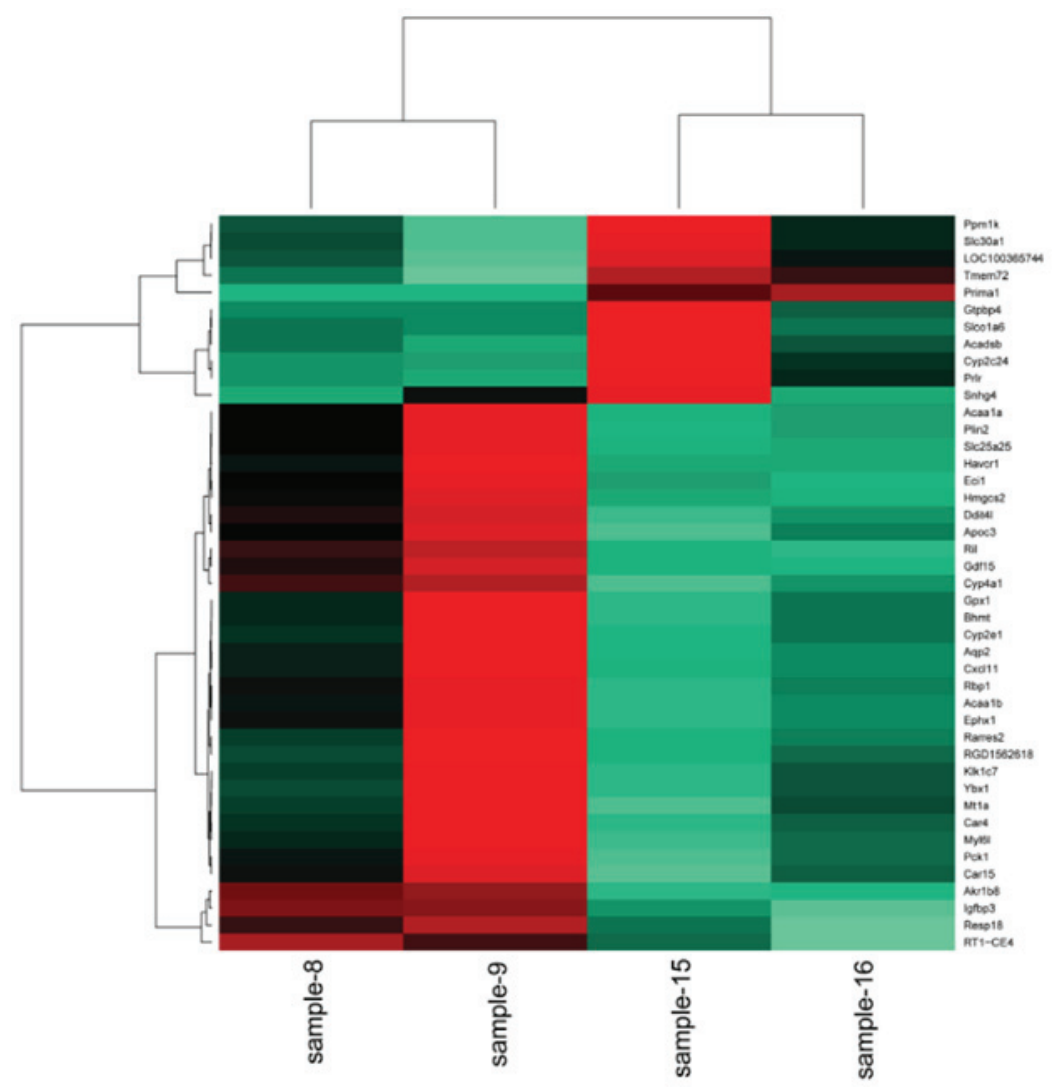

Figure 2. Hierarchical clustering of differentially expressed genes in the ginsenoside-Rg3 treatment group compared with the DN control group. Each row represents a differentially expressed gene, while each column represents a sample. In the heatmap, green represents genes that are downregulated and red represents genes that are upregulated. 
Table IV. KEGG enrichment analysis of differential expressed genes.

\begin{tabular}{|c|c|c|c|c|c|}
\hline Group & ID & Pathway & P-value & Count & Genes \\
\hline \multirow[t]{3}{*}{$\begin{array}{l}\text { Normal control vs. } \\
\text { DN control group }\end{array}$} & rno03320 & PPAR signaling pathway & $4.09253 \times 10^{-5}$ & 7 & $\begin{array}{l}\text { Acaa1a, Angpt14, Apoc3, } \\
\text { Cyp4a1,Cyp4a3,Hmgcs2,Pck1 }\end{array}$ \\
\hline & rno04978 & Mineral absorption & 0.00179 & 5 & Fth1, Mt1a, Mt2A, S100g, Tf \\
\hline & $\operatorname{rno} 00071$ & Fatty acid degradation & 0.00199 & 5 & $\begin{array}{l}\text { Acaa1a, Acaa2, Cyp4a1, } \\
\text { Cyp4a3, Eci1 }\end{array}$ \\
\hline \multirow{2}{*}{$\begin{array}{l}\text { DN control vs. } \\
\text { ginsenoside-Rg3 } \\
\text { treatment group }\end{array}$} & rno03320 & PPAR signaling pathway & $5.51233 \times 10^{-5}$ & 6 & $\begin{array}{l}\text { Acaa1a, Acaa1b, Apoc3, } \\
\text { Cyp4a1, Hmgcs2, Pck1 }\end{array}$ \\
\hline & rno00071 & Fatty acid degradation & 0.00908 & 4 & Acaa1a, Acaa1b, Cyp4a1, Eci1 \\
\hline
\end{tabular}

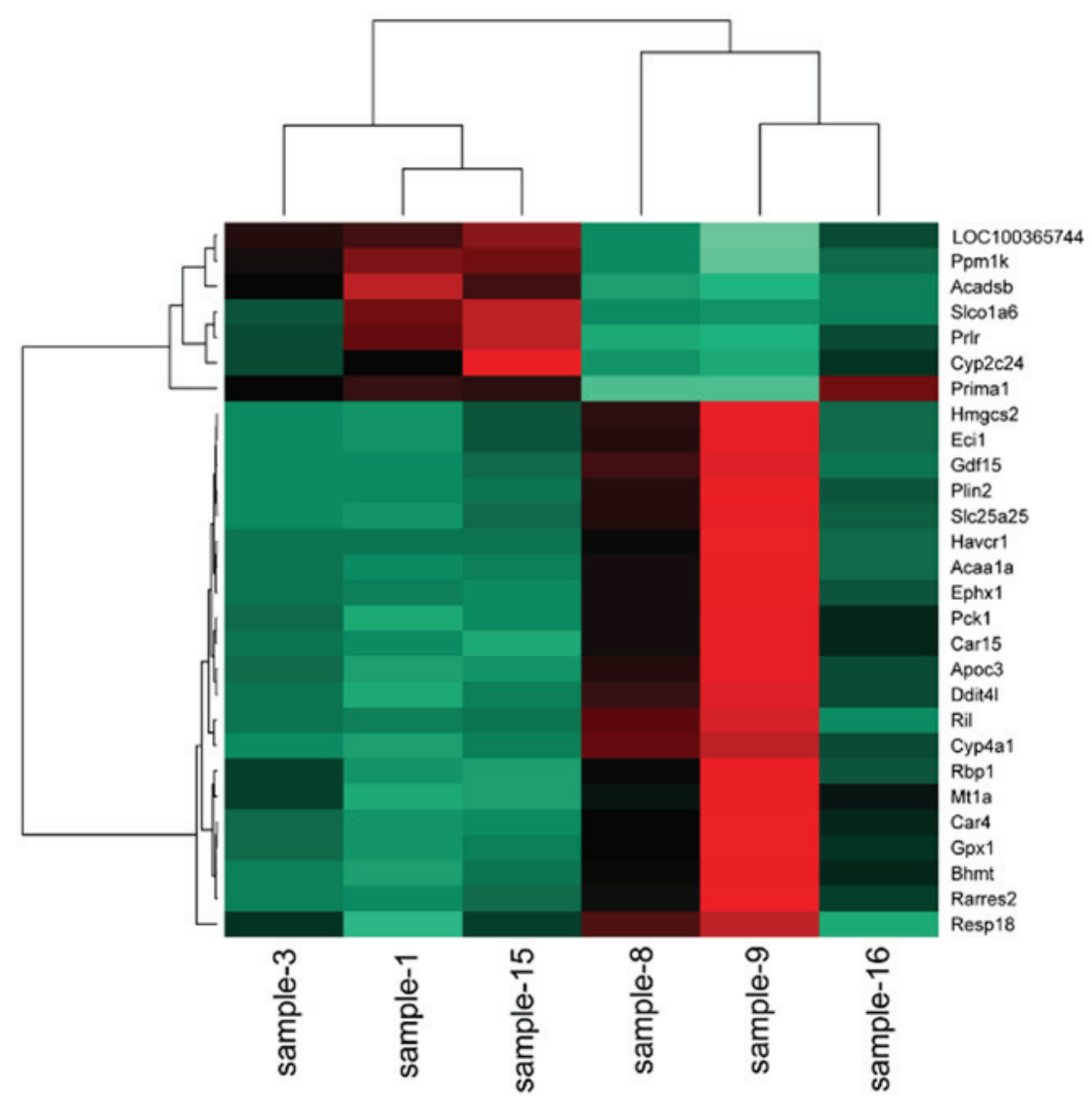

Figure 3. Hierarchical clustering of differentially expressed genes among six samples. Each row represents a differentially expressed gene, while each column represents a sample. In the heatmap, green represents genes that are downregulated red represents genes that are upregulated.

normal control group, were found to be upregulated in the ginsenoside-Rg3 treatment group compared with the DN control group. In addition, upregulation of 7 genes in the DN control group compared with the normal control group were downregulated in the ginsenoside- $\mathrm{Rg} 3$ treatment group compared with the DN control group. These 28 differentially expressed genes are shown in Table III.

Cluster analysis. To obtain a global view of the differentially expressed genes, the hierarchical cluster was performed among the samples. The heat maps indicated that the transcriptomes are different (Figs. 1-3).

$G O$ and KEGG analysis. KEGG analysis was performed on the differentially expressed genes in the DN control group compared with the normal control group and in the
ginsenoside-Rg3 treatment group compared with the DN control group, respectively. Significantly affected pathways were identified in the upregulated gene sets in the normal control compared with the DN control group, and also in the downregulated gene sets in the DN control compared with the ginsenoside-Rg3 treatment group (Table IV).

The pathways, including peroxisome PPAR signaling pathway, mineral absorption and fatty acid degradation, may be critical in ginsenoside- $\mathrm{Rg} 3$ treatment of $\mathrm{DN}$, implicating certain key genes enriched by these pathways (such as Acaala and Acaalb) as potential therapeutic targets.

To gain a greater understanding of the biological implications, GO enrichment analysis on differentially expressed genes was performed. GO is a standardized gene 
Table V. GO analysis on 28 differentially expressed genes.

\begin{tabular}{|c|c|c|c|c|}
\hline Ontology & GO ID & Term & P-value & Genes \\
\hline GO_BP & GO:0006641 & Triglyceride metabolic process & 0.00194 & GPX1, APOC3, PCK1 \\
\hline GO_BP & GO:0006639 & Acylglycerol metabolic process & 0.00242 & GPX1, APOC3, PCK1 \\
\hline GO_BP & GO:0006638 & Neutral lipid metabolic process & 0.00260 & GPX1, APOC3, PCK1 \\
\hline GO_BP & GO:0006720 & Isoprenoid metabolic process & 0.00260 & RARRES2, HMGCS2, RBP1 \\
\hline GO_BP & GO:0006662 & Glycerol ether metabolic process & 0.00269 & GPX1, APOC3, PCK1 \\
\hline GO_BP & GO:0018904 & Organic ether metabolic process & 0.00287 & GPX1, APOC3, PCK1 \\
\hline GO_BP & GO:0009636 & Response to toxin & 0.00335 & GPX1, MT1A, EPHX1 \\
\hline GO_BP & GO:0009725 & Response to hormone stimulus & 0.00380 & $\begin{array}{l}\text { GPX1, HMGCS2, APOC3, } \\
\text { CAR4, PCK1 }\end{array}$ \\
\hline GO_BP & GO:0010033 & Response to organic substance & 0.00563 & $\begin{array}{l}\text { GPX1, HMGCS2, APOC3, } \\
\text { EPHX1, CAR4, PCK1 }\end{array}$ \\
\hline GO_BP & GO:0009719 & Response to endogenous stimulus & 0.00576 & $\begin{array}{l}\text { GPX1, HMGCS2, APOC3, } \\
\text { CAR4, PCK1 }\end{array}$ \\
\hline GO_BP & GO:0046486 & Glycerolipid metabolic process & 0.01784 & GPX1, APOC3, PCK1 \\
\hline GO_MF & GO:0046914 & Transition metal ion binding & 0.02858 & $\begin{array}{l}\text { RIL, CYP4A1, MT1A, BHMT, } \\
\text { CYP2C24, CAR4, PCK1 }\end{array}$ \\
\hline GO_BP & GO:0007584 & Response to nutrient & 0.02963 & GPX1, HMGCS2, APOC3 \\
\hline GO_BP & GO:0043434 & Response to peptide hormone stimulus & 0.03400 & HMGCS2, APOC3, PCK1 \\
\hline GO_MF & GO:0009055 & Electron carrier activity & 0.03467 & ACADSB, CYP4A1, CYP2C24 \\
\hline GO_BP & GO:0009410 & Response to xenobiotic stimulus & 0.03516 & GPX1, EPHX1 \\
\hline GO_BP & GO:0016101 & Diterpenoid metabolic process & 0.03899 & RARRES2, RBP1 \\
\hline GO_BP & GO:0001523 & Retinoid metabolic process & 0.03899 & RARRES2, RBP1 \\
\hline GO_BP & GO:0006721 & Terpenoid metabolic process & 0.04281 & RARRES2, RBP1 \\
\hline GO_BP & GO:0055114 & Oxidation reduction & 0.04416 & $\begin{array}{l}\text { GPX1, ACADSB, CYP4A1, } \\
\text { CYP2C } 24\end{array}$ \\
\hline
\end{tabular}

GO_BP, biological process GO_MF, molecular function.

Table VI. KEGG enrichment analysis on 28 differentially expressed genes.

\begin{tabular}{lllll}
\hline Pathway & \multicolumn{1}{c}{ Term } & \multicolumn{1}{c}{ P-value } & Count & \multicolumn{1}{c}{ Genes } \\
\hline rno03320 & PPAR signaling pathway & $7.88382 \times 10^{-8}$ & 5 & Acaa1a,Apoc3,Cyp4a1,Hmgcs2,Pck1 \\
rno00071 & Fatty acid degradation & $4.42130 \times 10^{-5}$ & 3 & Acaa1a, Cyp4a1, Eci1 \\
rno00910 & Nitrogen metabolism & 0.00029 & 2 & Car15, Car4 \\
rno04964 & Proximal tubule bicarbonate reclamation & 0.00049 & 2 & Car4, Pck1 \\
rno00280 & Valine, leucine and isoleucine degradation & 0.00286 & 2 & Acaa1a, Hmgcs2
\end{tabular}

functional classification system, which provides a strictly defined concept and controlled vocabulary to describe properties of genes and their products. GO-annotated differentially expressed genes predominantly belong to the three functional clusters (biological process, cellular component and molecular function). Enrichment analysis focused on the 28 differentially expressed genes (21 upregulated genes and 7 downregulated genes) following administration of ginsenoside-Rg3. It was demonstrated that the 28 differentially expressed genes in the cluster of biological process was predominantly associated with lipid metabolism process (Table V). In addition, KEGG enrichment analysis was also performed on the 28 genes. Five pathways, including the
PPAR signaling pathway, fatty acid degradation, nitrogen metabolism, proximal tubule bicarbonate reclamation and valine, leucine and isoleucine degradation were shown to be enriched (Table VI).

Pathway analysis. Differentially expressed genes were mapped to rno00071 and rno03320 pathways of KEGG to observe the gene distribution and effects. In the rno00071 pathway, it was demonstrated that ginsenoside-Rg3 treatment reversed the expression of the acyl-CoA dehydrogenase, short/branched chain gene, acetyl-CoA acyltransferase, enoyl-CoA hydratase/3-hydroxyacyl CoA dehydrogenase and cytochrome P450 family 4 subfamily a polypeptide 2, suggesting a critical role of fatty acid metabolism pathway 
FATTY ACID DEGRADATION

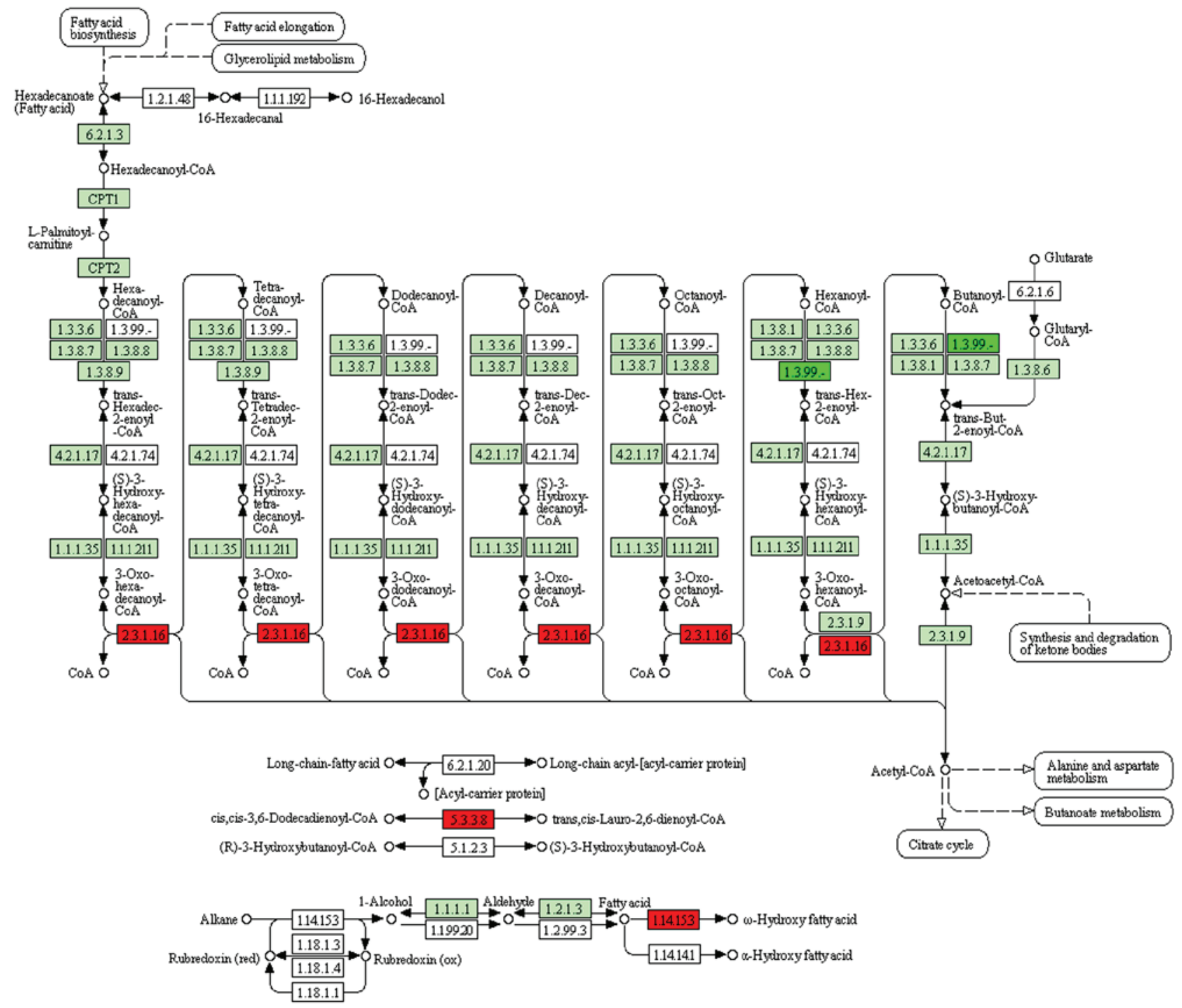

00071116015
(c) Kanehisa Laboratonies

Figure 4. Differentially expressed gene distribution in the rno00071 pathway. Differentially expressed genes in the DN control group compared with the normal control group were shown to be associated with fatty acid degradation. Bright green, upregulated genes; red, downregulated genes; pale green, species specific genes.

through affecting the four key genes in the process of DN with ginsenoside-Rg3 treatment (Figs. 4 and 5). In the rno03320 pathway, change in the expression of five genes: HMGCS2, APoCii, CYP4A1, ThinoleseB, PEPCK in DN was reversed by treatment with ginsenoside-Rg3 (Figs. 6 and 7).

\section{Discussion}

Recently, the revolution of next generation sequencing (NGS) has had a great impact on genome research. RNA sequencing (RNA-Seq) is an innovative NGS tool for the comprehensive transcriptome profiling on a genome-wide scale using deep-sequencing technologies $(11,12)$. Studies using this tool have already altered perception on the extent and complexity of eukaryotic transcriptomes. RNA-Seq also provides a far more precise measurement of levels of transcripts and their isoforms (11). In contrast to the technologies of microarray and qPCR analysis, RNA-seq allows for the identification of novel transcripts, examination of all RNA species, and identification of alternative splicing and mutations (13).

The present study collected renal cortex samples of six rats ( $\mathrm{n}=2 /$ group), which were classified into the normal control group, DN control group and ginsenoside- $\operatorname{Rg} 3$ treatment group. Gene expression profiling analysis was performed using an NGS strategy with the aim of identifying biomarker genes relevant to the molecular pathogenesis of DN. In total, there were 78 differentially expressed genes in the DN control group when compared with the normal control group, of which 52 genes were upregulated and 26 genes were downregulated. Expression of 43 genes was differentially regulated in the ginsenoside- $\operatorname{Rg} 3$ treatment group when compared with the DN control group, consisting 


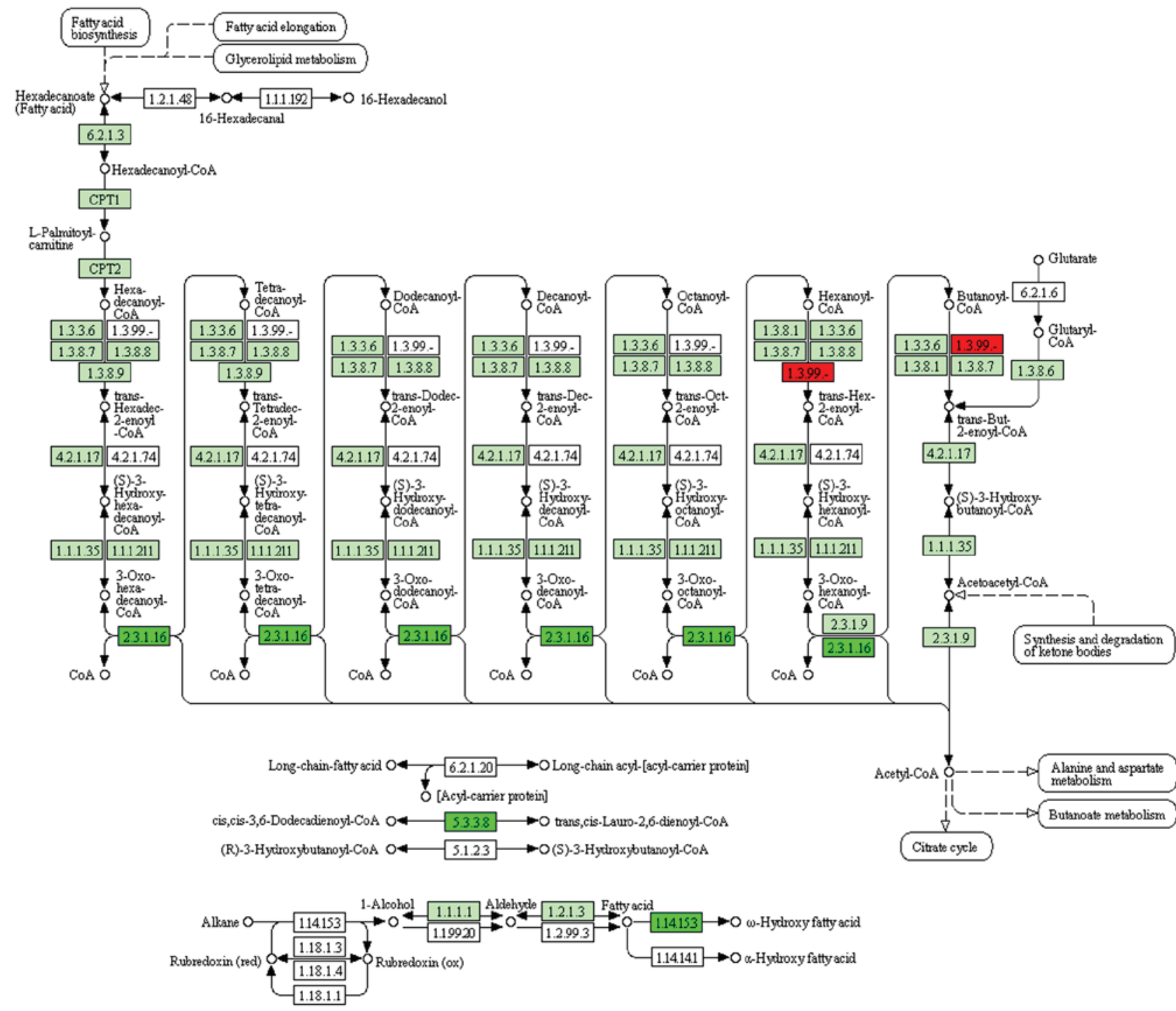

00071116015
(c) Kanehisa Laboratonies

Figure 5. Differentially expressed gene distribution in the rno00071 pathway. Differentially express genes in the ginsenoside-Rg3 treatment group compared with the DN control group were shown to be associated with fatty acid degradation. Bright green, upregulated genes; red, downregulated genes; pale green, species specific genes.

of 10 upregulated and 33 genes downregulated. Notably, 21 downregulated genes in the DN control group were upregulated in the ginsenoside- $\mathrm{Rg} 3$ treatment group, and 7 upregulated genes in the DN control group were downregulated in the ginsenoside- $\mathrm{Rg} 3$ treatment group. GO annotation analysis showed that the differentially expressed genes were predominantly associated with the lipid metabolism process. KEGG pathway enrichment analysis showed that fatty acid degradation and PPAR signaling pathways were associated with the differentially expressed genes.

Recently, lipid metabolism disorder has become a focus of research in the pathogenesis of DN. Disordered lipid metabolism and renal lipid accumulation are not only associated with obesity-related renal disease and DN, but they may also contribute to the disease process (14). Sustained hyperglycemia in diabetes promotes fatty acid (FA) synthesis and triacylglycerol (TG) accumulation. Elevated serum TG, free FAs (FFAs), and modified cholesterol cause ectopic lipid accumulation in nonadipose tissues, leading to lipotoxicity (15), which may be involved in the pathogenesis of DN (16). Herman-Edelstein et al (17) investigated the association of altered renal TG and cholesterol metabolism with lipid accumulation in patients with DN. The results showed a highly significant correlation between glomerular filtration rate, inflammation and lipid metabolism associated genes, suggesting a potential role of abnormal lipid metabolism in the pathogenesis of DN (17).

PPAR is a member of the nuclear hormone receptor superfamily and is critical in lipid metabolism (18). PPAR is highly expressed in various organs, such as the liver, renal cortex and heart. Knocking out PPAR $\alpha$ appeared to aggravate the severity of DN through an increase in extracellular matrix 


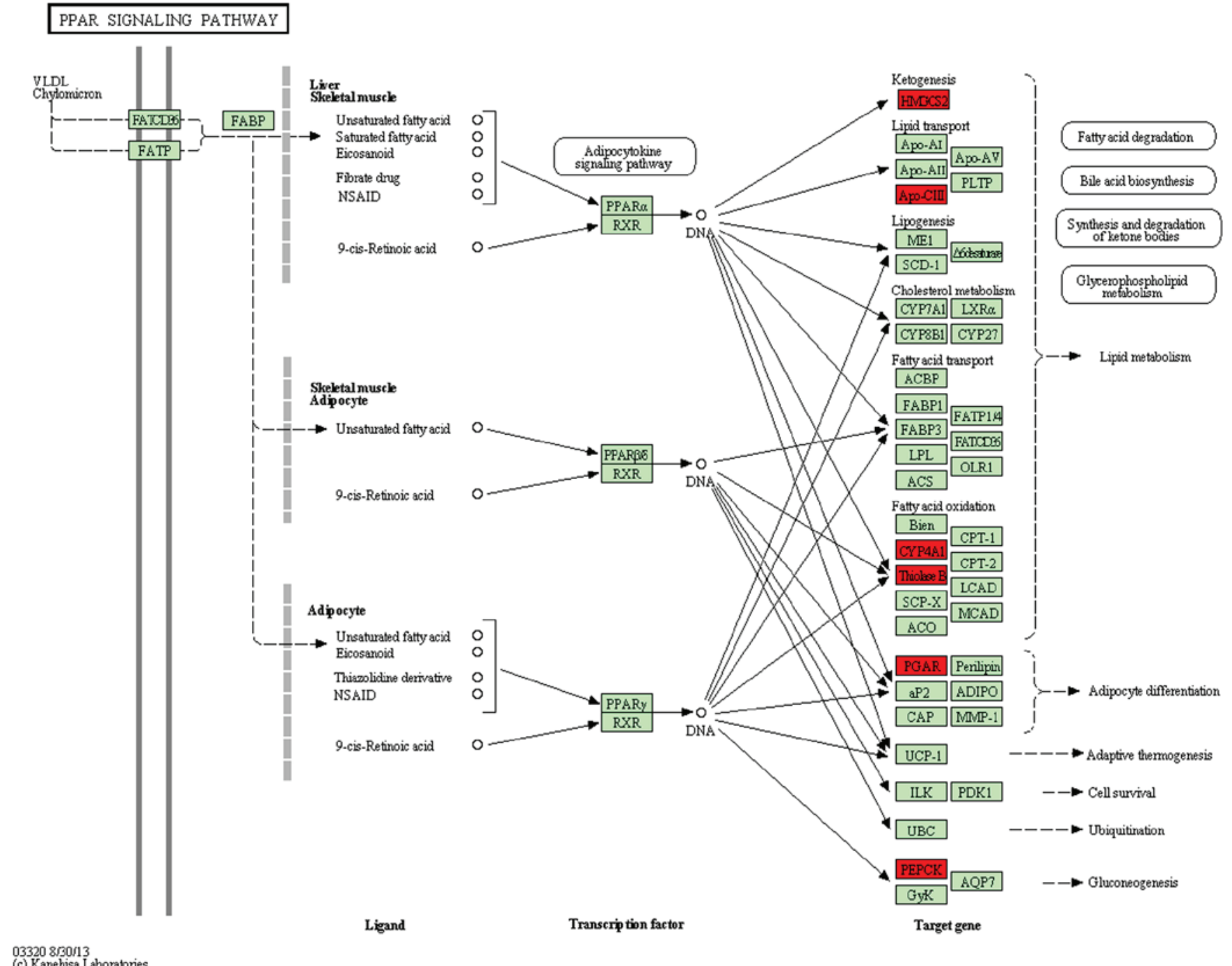

Figure 6. Differentially expressed gene distribution in the rno03320 pathway. Differentially express genes in the DN control group compared with the normal control group were shown to be associated with the PPAR signaling pathway. Red, downregulated genes; pale green, species specific genes. PPAR, peroxisome proliferator-activated receptor.

formation, inflammation, and circulating FFA and TG concentrations (19). PPAR- $\gamma$ is the most extensively studied PPAR subtype and is involved in adipocyte differentiation, and glucose and lipid metabolism. The mechanisms of PPAR- $\gamma$ in DN remain to be fully elucidated. A study suggested PPAR- $\gamma$ has an important role in regulating insulin sensitivity (20). Gene polymorphisms of PPAR- $\gamma$ gene polymorphism Ala12 carriers exhibited an improvement in insulin sensitivity (21), and may be responsible for the development of DN.

Current therapeutic strategies for DN remain suboptimal and are directed at delaying disease progression, for example, intensive glucose and blood pressure control, dyslipidemia and lipid-lowering drugs. Ginsenoside $\mathrm{Rg} 3$, an active component of Panax ginseng, has been identified to have a protective effect against hyperglycemia, obesity and diabetes in vivo (22). Animal experiments demonstrated the effect of ginsenoside Rg3 on diabetic renal damage (23). However, the precise mechanisms of these actions remain to be fully elucidated. Hwang et al (24) investigated the molecular basis of ginsenoside $\mathrm{Rg} 3$. Their results found that the effect of ginsenoside Rg3 in inhibiting adipocyte differentiation, and also PPAR- $\gamma$ signaling was involved in the inhibition of adipocyte differentiation by ginsenoside $\operatorname{Rg} 3$ (24). Ginsenoside has been shown to exhibit anti-obesity and anti-hyperglycemia effects that involve the PPAR mediated pathway (25). Sun et al (26) investigated the effect of ginsenoside Rg3 on the expression of VEGF and TNF- $\alpha$ in the retina of diabetic rats, and demonstrated that ginsenoside Rg3 could downregulate the expression of VEGF and TNF- $\alpha$, which may disrupt the development of diabetic retinopathy. Kang et al (22) analyzed the effect of ginsenoside $\mathrm{Rg} 3$ on the progression of renal disease in type II diabetic rat models, and provided evidence that $\mathrm{Rg} 3$ can prevent the progression of renal damage and dysfunction of diabetic rats. Lee et al (27) evaluated the effects of ginsenoside $\mathrm{Rg} 3$ on glucose uptake and the glucose transport system in mature 3T3-L1 cells, and demonstrated that ginsenoside Rg3 may stimulate the expression of insulin receptor substrate expression and phosphatidylinositol 3-kinase-110a protein, which may therefore be a valuable antidiabetic and antihyperglycemic agent. Bu et al (15) also demonstrated similar effects of ginsenoside Rg3 in reducing the fasting blood glucose level, reducing food and water intake, improving oral glucose tolerance, and repairing injured pancreas 


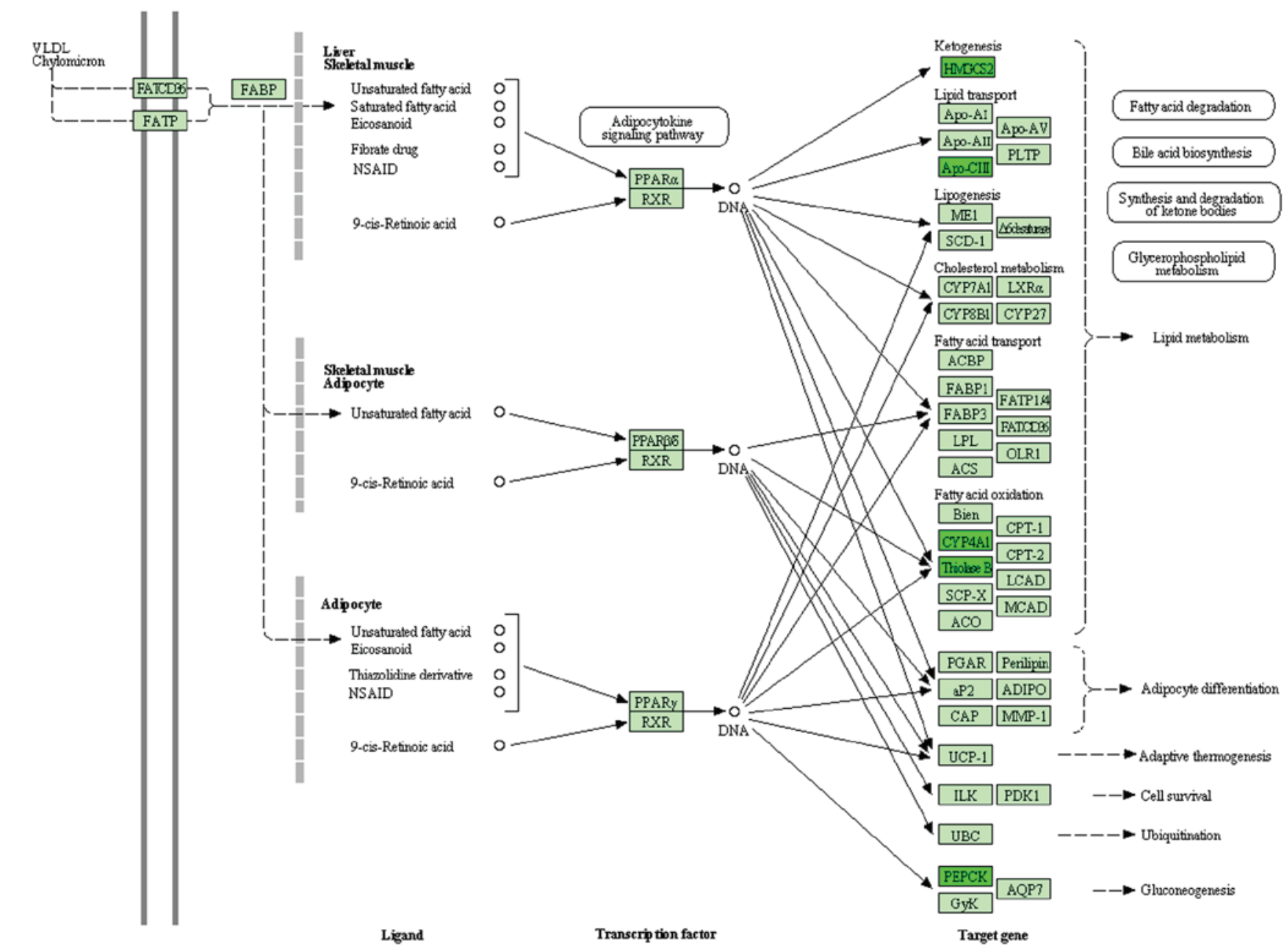

03320 8/30/13
(c) Kanehisa Laboratories

Figure 7. Differentially expressed gene distribution in rno03320 pathway. Differentially expressed genes in the in the ginsenoside-Rg3 treatment group compared with the DN control group were shown to be associated with the PPAR signaling pathway. Bright green, upregulated genes; pale green, species specific genes. PPAR, peroxisome proliferator-activated receptor.

tissues of alloxan-induced diabetic mice. These studies suggest that ginsenoside $\mathrm{Rg} 3$ has potential for clinical use in preventing and treating diabetes and its complications. The present study also suggested that ginsenoside $\operatorname{Rg} 3$ may be used as a novel and useful adjunctive drug for the treatment of DN.

High throughput RNA-Seq technology allows comprehensive transcriptome profiling. A set genes were identified to be differentially expressed following ginsenoside Rg3 treatment. Gene set enrichment analyses identified the specific biological processes, predominantly lipid/fatty acid metabolism and the PPAR signaling pathway, were associated with these genes. The identification of these genes and pathway analyses have provided novel insights into the molecular mechanisms underlying the effect of ginsenoside-Rg3 on DN. As the sample sizes in the present study are small, these findings require further validation.

\section{Acknowledgements}

This study was supported by the Enterprise postdoctoral fund in Liaoning province (grant no. 106561).

\section{References}

1. Vinik AI, Nevoret ML, Casellini C and Parson H: Diabetic neuropathy. Endocrinol Metab Clin North Am 42: 747-787, 2013.

2. Waanders F, Visser FW and Gans RO: Current concepts in the management of diabetic nephropathy. Neth J Med 71: 448-458, 2013.

3. Zeng CC, Liu X, Chen GR, Wu QJ, Liu WW, Luo HY and Cheng JG: The molecular mechanism of rhein in diabetic nephropathy. Evid Based Complement Alternat Med 2014: 487097, 2014.

4. Yin J, Zhang $\mathrm{H}$ and Ye J: Traditional chinese medicine in treatment of metabolic syndrome. Endocr Metab Immune Disord Drug Targets 8: 99-111, 2008.

5. Lee JH, Choi SH, Kwon OS, Shin TJ, Lee JH, Lee BH, Yoon IS, Pyo MK, Rhim H, Lim YH, et al: Effects of ginsenosides, active ingredients of panax ginseng, on development, growth and life span of caenorhabditis elegans. Biol Pharm Bull 30: 2126-2134, 2007.

6. Trapnell C, Pachter L and Salzberg SL: TopHat: Discovering splice junctions with RNA-Seq. Bioinformatics 25: 1105-1111, 2009.

7. Trapnell C, Williams BA, Pertea G, Mortazavi A, Kwan G, van Baren MJ, Salzberg SL, Wold BJ and Pachter L: Transcript assembly and quantification by RNA-Seq reveals unannotated transcripts and isoform switching during cell differentiation. Nat Biotechnol 28: 511-515, 2010. 
8. Kanehisa M, Araki M, Goto S, Hattori M, Hirakawa M, Itoh M, Katayama T, Kawashima S, Okuda S, Tokimatsu T and Yamanishi Y: KEGG for linking genomes to life and the environment. Nucleic Acids Res 36: D480-D484, 2008.

9. Kanehisa M, Goto S, Sato Y, Furumichi M and Tanabe M: KEGG for integration and interpretation of large-scale molecular data sets. Nucleic Acids Res 40: D109-D114, 2012.

10. Chen YA, Tripathi LP and Mizuguchi K: TargetMine, an integrated data warehouse for candidate gene prioritisation and target discovery. PLoS One 6: e17844, 2011.

11. Wang Z, Gerstein M and Snyder M: RNA-Seq: A revolutionary tool for transcriptomics. Nat Rev Genet 10: 57-63, 2009.

12. Sutherland GT, Janitz M and Kril JJ: Understanding the pathogenesis of Alzheimer's disease: Will RNA-Seq realize the promise of transcriptomics? J Neurochem 116: 937-946, 2011.

13. Satoh J, Yamamoto Y, Asahina N, Kitano S and Kino Y: RNA-Sec data mining: Downregulation of NeuroD6 serves as a possible biomarker for Alzheimer's disease brains. Dis Markers 2014 123165,2014

14. de Vries AP, Ruggenenti P, Ruan XZ, Praga M, Cruzado JM, Bajema IM, D'Agati VD, Lamb HJ, Pongrac Barlovic D, Hojs R, et al: Fatty kidney: Emerging role of ectopic lipid in obesity-related renal disease. Lancet Diabetes Endocrinol 2: 417-426, 2014.

15. Bu QT, Zhang WY, Chen QC, Zhang CZ, Gong XJ, Liu WC, $\mathrm{Li} W$ and Zheng YN: Anti-diabetic effect of ginsenoside $\mathrm{Rb}(3)$ in alloxan-induced diabetic mice. Med Chem 8: 934-941, 2012.

16. Jiang T, Wang XX, Scherzer P, Wilson P, Tallman J, Takahashi H, Li J, Iwahashi M, Sutherland E, Arend L and Levi M: Farnesoid X receptor modulates renal lipid metabolism, fibrosis and diabetic nephropathy. Diabetes 56: 2485-2493, 2007.

17. Herman-Edelstein M, Scherzer P, Tobar A, Levi M and Gafter U: Altered renal lipid metabolism and renal lipid accumulation in human diabetic nephropathy. J Lipid Res 55: 561-572, 2014.

18. Isselmann I and Green S: Activation of a member of the steroid hormone receptor superfamily by peroxisome proliferators. Nature 347: 645-650, 1990.
19. Park CW, Kim HW, Ko SH, Chung HW, Lim SW, Yang CW, Chang YS, Sugawara A, Guan Y and Breyer MD: Accelerated diabetic nephropathy in mice lacking the peroxisome proliferator-activated receptor alpha. Diabetes 55: 885-893, 2006.

20. Duan SZ, Ivashchenko CY, Whitesall SE, D'Alecy LG, Duquaine DC, Brosius FC III, Gonzalez FJ, Vinson C, Pierre MA, Milstone DS and Mortensen RM: Hypotension, lipodystrophy and insulin resistance in generalized PPARgamma-deficient mice rescued from embryonic lethality. J Clin Invest 117: 812-822, 2007.

21. Deeb SS, Fajas L, Nemoto M, Pihlajamäki J, Mykkänen L, Kuusisto J, Laakso M, Fujimoto W and Auwerx J: A Pro12Ala substitution in PPARgamma2 associated with decreased receptor activity, lower body mass index and improved insulin sensitivity. Nat Genet 20: 284-287, 1998

22. Kang KS, Yamabe N, Kim HY, Park JH and Yokozawa T: Therapeutic potential of $20(\mathrm{~S})$-ginsenoside $\operatorname{Rg}(3)$ against streptozotocin-induced diabetic renal damage in rats. Eur J Pharmacol 591: 266-272, 2008.

23. Kim M, Ahn BY, Lee JS, Chung SS, Lim S, Park SG, Jung HS, Lee HK and Park KS: The ginsenoside Rg3 has a stimulatory effect on insulin signaling in L6 myotubes. Biochem Biophys Res Commun 389: 70-73, 2009.

24. Hwang JT, Lee MS, Kim HJ, Sung MJ, Kim HY, Kim MS and Kwon DY: Antiobesity effect of ginsenoside Rg3 involves the AMPK and PPAR-gamma signal pathways. Phytother Res 23: 262-266, 2009.

25. Ni HX, Yu NJ and Yang XH: The study of ginsenoside on PPARgamma expression of mononuclear macrophage in type 2 diabetes. Mol Biol Rep 37: 2975-2979, 2010.

26. Sun HQ and Zhou ZY: Effect of ginsenoside-Rg3 on the expression of VEGF and TNF- $\alpha$ in retina with diabetic rats. Int J Ophthalmol 3: 220-223, 2010.

27. Lee OH, Lee HH, Kim JH and Lee BY: Effect of ginsenosides $\mathrm{Rg} 3$ and $\mathrm{Re}$ on glucose transport in mature 3T3-L1 adipocytes. Phytother Res 25: 768-773, 2011. 